\title{
TENSOR EXTENSION PROPERTIES OF $C(K)$-REPRESENTATIONS AND APPLICATIONS TO UNCONDITIONALITY
}

\author{
CHRISTOPH KRIEGLER and CHRISTIAN LE MERDY ${ }^{凶}$
}

(Received 8 January 2009; accepted 10 July 2009)

Communicated by A. M. Hassell

\begin{abstract}
Let $K$ be any compact set. The $C^{*}$-algebra $C(K)$ is nuclear and any bounded homomorphism from $C(K)$ into $B(H)$, the algebra of all bounded operators on some Hilbert space $H$, is automatically completely bounded. We prove extensions of these results to the Banach space setting, using the key concept of $R$-boundedness. Then we apply these results to operators with a uniformly bounded $H^{\infty}$-calculus, as well as to unconditionality on $L^{p}$. We show that any unconditional basis on $L^{p}$ 'is' an unconditional basis on $L^{2}$ after an appropriate change of density.
\end{abstract}

2000 Mathematics subject classification: primary 47A60; secondary 46B28.

Keywords and phrases: functional calculus, $R$-boundedness, unconditionality, tensor products.

\section{Introduction}

Throughout this paper, we let $K$ be a nonempty compact set and we let $C(K)$ be the algebra of all continuous functions $f: K \rightarrow \mathbb{C}$, equipped with the supremum norm. A representation of $C(K)$ on some Banach space $X$ is a bounded unital homomorphism $u: C(K) \rightarrow B(X)$ into the algebra $B(X)$ of all bounded operators on $X$. Such representations appear naturally and play a major role in several fields of operator theory, including functional calculi, spectral theory and spectral measures, and the classification of $C^{*}$-algebras. Several recent papers, in particular [8, 12, 21, 23], have emphasized the rich and fruitful interplays between the notion of $R$-boundedness, unconditionality and various functional calculi. The aim of this paper is to establish new properties of the $C(K)$-representations involving $R$-boundedness, and to give applications to $H^{\infty}$ calculus (in the sense of $[6,21])$ and to unconditionality in $L^{p}$-spaces.

We recall the definition of $R$-boundedness (see [2,4]). Let $\left(\epsilon_{k}\right)_{k \geq 1}$ be a sequence of independent Rademacher variables on some probability space $\Omega_{0}$. That is, the $\epsilon_{k}$

The first author is supported by the Karlsruhe House of Young Scientists and the Franco-German University DFH-UFA, the second author is supported by the research program ANR-06-BLAN-0015.

(C) 2010 Australian Mathematical Publishing Association Inc. 1446-7887/2010 \$16.00 
take values in the set $\{-1,1\}$ and $\operatorname{Prob}\left(\left\{\epsilon_{k}=1\right\}\right)=\operatorname{Prob}\left(\left\{\epsilon_{k}=-1\right\}\right)=1 / 2$. For any Banach space $X$, we let $\operatorname{Rad}(X) \subset L^{2}\left(\Omega_{0} ; X\right)$ be the closure of $\operatorname{Span}\left\{\epsilon_{k} \otimes x: k \geq 1\right.$, $x \in X\}$ in $L^{2}\left(\Omega_{0} ; X\right)$. Thus, for all $x_{1}, \ldots, x_{n}$ in $X$,

$$
\left\|\sum_{k} \epsilon_{k} \otimes x_{k}\right\|_{\operatorname{Rad}(X)}=\left(\int_{\Omega_{0}}\left\|\sum_{k} \epsilon_{k}(\lambda) x_{k}\right\|_{X}^{2} d \lambda\right)^{1 / 2} .
$$

By definition, a set $\tau \subseteq B(X)$ is $R$-bounded if there is a constant $C \geq 0$ such that, for all finite families $T_{1}, \ldots, T_{n}$ in $\tau$, and $x_{1}, \ldots, x_{n}$ in $X$,

$$
\left\|\sum_{k} \epsilon_{k} \otimes T_{k} x_{k}\right\|_{\operatorname{Rad}(X)} \leq C\left\|\sum_{k} \epsilon_{k} \otimes x_{k}\right\|_{\operatorname{Rad}(X)} .
$$

In this case, we let $R(\tau)$ denote the smallest possible $C$. It is called the $R$-bound of $\tau$. By convention, we write $R(\tau)=\infty$ if $\tau$ is not $R$-bounded.

It will be convenient to let $\operatorname{Rad}_{n}(X)$ denote the subspace of $\operatorname{Rad}(X)$ of all finite sums $\sum_{k=1}^{n} \epsilon_{k} \otimes x_{k}$. If $X=H$ is a Hilbert space, then $\operatorname{Rad}_{n}(H)=\ell_{n}^{2}(H)$ isometrically and all bounded subsets of $B(H)$ are automatically $R$-bounded. Conversely, if $X$ is not isomorphic to a Hilbert space, then $B(X)$ contains bounded subsets which are not $R$-bounded [1, Proposition 1.13].

In order to provide motivation for the results in this paper, we recall two wellknown properties of $C(K)$-representations on the Hilbert space $H$. First, any bounded homomorphism $u: C(K) \rightarrow B(H)$ is completely bounded, and $\|u\|_{\mathrm{cb}} \leq\|u\|^{2}$, that is for all integers $n \geq 1$, the tensor extension $I_{M_{n}} \otimes u: M_{n}(C(K)) \rightarrow M_{n}(B(H))$ satisfies $\left\|I_{M_{n}} \otimes u\right\| \leq\|u\|^{2}$ when $M_{n}(C(K))$ and $M_{n}(B(H))$ are both equipped with their natural $C^{*}$-algebra norms. This in turn implies that any bounded homomorphism $u: C(K) \rightarrow B(H)$ is similar to a $*$-representation, a result going back at least to [3]. We refer to $[28,30]$ and the references therein for some information on completely bounded maps and similarity properties.

Second, let $u: C(K) \rightarrow B(H)$ be a bounded homomorphism. Then for all $b_{1}, \ldots$, $b_{n}$ lying in the commutant of the range of $u$ and for all $f_{1}, \ldots, f_{n}$ in $C(K)$,

$$
\left\|\sum_{k} u\left(f_{k}\right) b_{k}\right\| \leq\|u\|^{2} \sup _{t \in K}\left\|\sum_{k} f_{k}(t) b_{k}\right\| .
$$

This property is essentially a rephrasing of the fact that $C(K)$ is a nuclear $C^{*}$ algebra. More precisely, nuclearity means that the above property holds true for *-representations (see, for example, [19, Ch. 11] or [28, Ch. 12]), and its extension to arbitrary bounded homomorphisms easily follows from the similarity property mentioned above (see [25] for more explanations and developments).

Now let $X$ be a Banach space and let $u: C(K) \rightarrow B(X)$ be a bounded homomorphism. In Section 2, we will show the following analog of (1.1):

$$
\left\|\sum_{k} u\left(f_{k}\right) b_{k}\right\| \leq\|u\|^{2} R\left(\left\{\sum_{k} f_{k}(t) b_{k}: t \in K\right\}\right),
$$

provided that the $b_{k}$ commute with the range of $u$. 
Section 3 is devoted to the sectorial operators $A$ which have a uniformly bounded $H^{\infty}$-calculus, in the sense that they satisfy an estimate

$$
\|f(A)\| \leq C \sup _{t>0}|f(t)|
$$

for all bounded analytic functions $f$ on a sector $\Sigma_{\theta}$ surrounding $(0, \infty)$. Such operators turn out to have a natural $C(K)$-functional calculus. Applying (1.2) to the resulting representation $u: C(K) \rightarrow B(X)$, we show that (1.3) can be automatically extended to operator-valued analytic functions $f$ taking their values in the commutant of $A$. This is an analog of a remarkable result of Kalton and Weis [21, Theorem 4.4] which says that if an operator $A$ has a bounded $H^{\infty}$-calculus and $f$ is an operatorvalued analytic function taking its values in an $R$-bounded subset of the commutant of $A$, then the operator $f(A)$ arising from 'generalized $H^{\infty}$-calculus' is bounded.

In Section 4, we introduce matricially $R$-bounded maps $C(K) \rightarrow B(X)$, a natural analog of completely bounded maps in the Banach space setting. We show that if $X$ has property $(\alpha)$, then any bounded homomorphism $C(K) \rightarrow B(X)$ is automatically matricially $R$-bounded. This extends both the Hilbert space result mentioned above, and a result of de Pagter and Ricker [8, Corollary 2.19] which says that any bounded homomorphism $C(K) \rightarrow B(X)$ maps the unit ball of $C(K)$ into an $R$-bounded set, provided that $X$ has property $(\alpha)$.

In Section 5 , we give an application of matricial $R$-boundedness to the case when $X=L^{p}$. A classical result of Johnson and Jones [18] asserts that any bounded operator $T: L^{p} \rightarrow L^{p}$ acts, after an appropriate change of density, as a bounded operator on $L^{2}$. We show versions of this theorem for bases (more generally, for finite-dimensional decompositions). Indeed, we show that any unconditional basis (any $R$-basis) on $L^{p}$ becomes an unconditional basis (respectively a Schauder basis) on $L^{2}$ after an appropriate change of density. These results rely on Simard's extensions of the Johnson-Jones theorem established in [32].

We end this introduction with a few preliminaries and some notation. For any Banach space $Z$, we denote by $C(K ; Z)$ the space of all continuous functions $f: K \rightarrow$ $Z$, equipped with the supremum norm

$$
\|f\|_{\infty}=\sup \left\{\|f(t)\|_{Z}: t \in K\right\} .
$$

We may regard $C(K) \otimes Z$ as a subspace of $C(K ; Z)$ by identifying $\sum_{k} f_{k} \otimes z_{k}$ with the function $t \mapsto \sum_{k} f_{k}(t) z_{k}$, for all finite families $\left(f_{k}\right)_{k}$ in $C(K)$ and $\left(z_{k}\right)_{k}$ in $Z$. Moreover, $C(K) \otimes Z$ is dense in $C(K ; Z)$. Note that, for all integers $n \geq 1$, $C\left(K ; M_{n}\right)$ coincides with the $C^{*}$-algebra $M_{n}(C(K))$ mentioned above.

We will need the so-called 'contraction principle' which says that, for all $x_{1}, \ldots$, $x_{n}$ in a Banach space $X$ and all $\alpha_{1}, \ldots, \alpha_{n}$ in $\mathbb{C}$,

$$
\left\|\sum_{k} \epsilon_{k} \otimes \alpha_{k} x_{k}\right\|_{\operatorname{Rad}(X)} \leq 2 \sup _{k}\left|\alpha_{k}\right|\left\|\sum_{k} \epsilon_{k} \otimes x_{k}\right\|_{\operatorname{Rad}(X)} .
$$


We also recall that any unital commutative $C^{*}$-algebra is a $C(K)$-space (see, for example, [19, Ch. 4]). Thus our results concerning $C(K)$-representations apply as well to all these algebras. For example, we will apply them to $\ell^{\infty}$ in Section 5.

We let $I_{X}$ denote the identity mapping on a Banach space $X$, and we let $\chi_{B}$ denote the indicator function of a set $B$. If $X$ is a dual Banach space, we let $w^{*} B(X) \subseteq B(X)$ be the subspace of all $w^{*}$-continuous operators on $X$.

\section{The extension theorem}

Let $X$ be an arbitrary Banach space. For any compact set $K$ and any bounded homomorphism $u: C(K) \rightarrow B(X)$, we denote by

$$
E_{u}=\{b \in B(X): b u(f)=u(f) b \forall f \in C(K)\}
$$

the commutant of the range of $u$.

Our main purpose in this section is to prove (1.1). We start with the case when $C(K)$ is finite-dimensional.

Proposition 2.1. Let $N \geq 1$ and let $u: \ell_{N}^{\infty} \rightarrow B(X)$ be a bounded homomorphism. Let $\left(e_{1}, \ldots, e_{N}\right)$ be the canonical basis of $\ell_{N}^{\infty}$ and set $p_{i}=u\left(e_{i}\right), i=1, \ldots, N$. Then, for all $b_{1}, \ldots, b_{N} \in E_{u}$,

$$
\left\|\sum_{i=1}^{N} p_{i} b_{i}\right\| \leq\|u\|^{2} R\left(\left\{b_{1}, \ldots, b_{N}\right\}\right)
$$

PROOF. Since $u$ is multiplicative, each $p_{i}$ is a projection and $p_{i} p_{j}=0$ when $i \neq j$. Hence for all choices of signs $\left(\alpha_{1}, \ldots, \alpha_{N}\right) \in\{-1,1\}^{N}$,

$$
\sum_{i=1}^{N} p_{i} b_{i}=\sum_{i, j=1}^{N} \alpha_{i} \alpha_{j} p_{i} p_{j} b_{j}
$$

Furthermore,

$$
\left\|\sum_{i} \alpha_{i} p_{i}\right\|=\left\|u\left(\alpha_{1}, \ldots, \alpha_{N}\right)\right\| \leq\|u\|\left\|\left(\alpha_{1}, \ldots, \alpha_{N}\right)\right\|_{\ell_{N}^{\infty}}=\|u\| .
$$

Therefore, for all $x \in X$, we have the following chain of inequalities which prove the desired estimate:

$$
\begin{aligned}
\left\|\sum_{i} p_{i} b_{i} x\right\|^{2} & =\int_{\Omega_{0}}\left\|\sum_{i} \epsilon_{i}(\lambda) p_{i} \sum_{j} \epsilon_{j}(\lambda) p_{j} b_{j} x\right\|^{2} d \lambda \\
& \leq \int_{\Omega_{0}}\left\|\sum_{i} \epsilon_{i}(\lambda) p_{i}\right\|^{2}\left\|\sum_{j} \epsilon_{j}(\lambda) p_{j} b_{j} x\right\|^{2} d \lambda \\
& \leq\|u\|^{2} \int_{\Omega_{0}}\left\|\sum_{j} \epsilon_{j}(\lambda) b_{j} p_{j} x\right\|^{2} d \lambda
\end{aligned}
$$




$$
\begin{aligned}
& \leq\|u\|^{2} R\left(\left\{b_{1}, \ldots, b_{N}\right\}\right)^{2} \int_{\Omega_{0}}\left\|\sum_{j} \epsilon_{j}(\lambda) p_{j} x\right\|^{2} d \lambda \\
& \leq\|u\|^{4} R\left(\left\{b_{1}, \ldots, b_{N}\right\}\right)^{2}\|x\|^{2} .
\end{aligned}
$$

This concludes the proof.

The study of infinite-dimensional $C(K)$-spaces requires the use of second duals and $w^{*}$-topologies. We recall a few well-known facts that will be used later on in this paper. According to the Riesz representation theorem, the dual space $C(K)^{*}$ can be naturally identified with the space $M(K)$ of Radon measures on $K$. Next, the second dual space $C(K)^{* *}$ is a commutative $C^{*}$-algebra for the so-called Arens product. This product extends the product on $C(K)$ and is separately $w^{*}$-continuous, which means that, for all $\xi \in C(K)^{* *}$, the two linear maps

$$
v \in C(K)^{* *} \longmapsto v \xi \in C(K)^{* *} \text { and } v \in C(K)^{* *} \longmapsto \xi \nu \in C(K)^{* *}
$$

are $w^{*}$-continuous.

Equip the space $\mathcal{B}^{\infty}(K)$ of all bounded, Borel measurable functions from $K$ to $\mathbb{C}$ with the supremum norm. According to the duality pairing

$$
\langle f, \mu\rangle=\int_{K} f(t) d \mu(t) \quad \forall \mu \in M(K), f \in \mathcal{B}^{\infty}(K),
$$

one can regard $\mathcal{B}^{\infty}(K)$ as a closed subspace of $C(K)^{* *}$. Moreover, the restriction of the Arens product to $\mathcal{B}^{\infty}(K)$ coincides with the pointwise product. Thus we have the natural $C^{*}$-algebra inclusions

$$
C(K) \subseteq \mathcal{B}^{\infty}(K) \subseteq C(K)^{* *}
$$

See, for example, [7, pp. 366-367] and [5, Section 9] for further details.

Let $\widehat{\otimes}$ denote the projective tensor product on Banach spaces. We recall that, for any two Banach spaces $Y_{1}, Y_{2}$, we have a natural identification

$$
\left(Y_{1} \widehat{\otimes} Y_{2}\right)^{*} \simeq B\left(Y_{2}, Y_{1}^{*}\right)
$$

see, for example, [10, Section VIII.2]. This implies that when $X$ is a dual Banach space, $X=\left(X_{*}\right)^{*}$ say, then $B(X)=\left(X_{*} \widehat{\otimes} X\right)^{*}$ is a dual space. The next two lemmas are elementary.

LeMma 2.2. Let $X=\left(X_{*}\right)^{*}$ be a dual space, $S \in B(X)$, and define the right and left multiplication operators $R_{S}, L_{S}: B(X) \rightarrow B(X)$ by $R_{S}(T)=T S$ and $L_{S}(T)=S T$, respectively. Then $R_{S}$ is $w^{*}$-continuous whereas $L_{S}$ is $w^{*}$-continuous if (and only if) $S$ is $w^{*}$-continuous.

PROOF. The tensor product mapping $I_{X_{*}} \otimes S$ on $X_{*} \otimes X$ uniquely extends to a bounded map $r_{S}: X_{*} \widehat{\otimes} X \rightarrow X_{*} \widehat{\otimes} X$, and we have $R_{S}=r_{S}^{*}$. Thus $R_{S}$ is $w^{*}$-continuous. 
Likewise, if $S$ is $w^{*}$-continuous and if we let $S_{*}: X_{*} \rightarrow X_{*}$ be its pre-adjoint map, the tensor product mapping $S_{*} \otimes I_{X}$ on $X_{*} \otimes X$ extends to a bounded map $l_{S}: X_{*} \widehat{\otimes} X \rightarrow$ $X_{*} \widehat{\otimes} X$, and $L_{S}=l_{S}^{*}$. Thus $L_{S}$ is $w^{*}$-continuous. The converse (which we will not use) is left to the reader.

Lemma 2.3. Let $u: C(K) \rightarrow B(X)$ be a bounded map. Suppose that $X$ is a dual space. Then there exists a (necessarily unique) $w^{*}$-continuous linear mapping $\tilde{u}$ : $C(K)^{* *} \rightarrow B(X)$ whose restriction to $C(K)$ coincides with $u$. Moreover, $\|\widetilde{u}\|=\|u\|$.

Furthermore, if $u$ is a homomorphism and $u$ takes values in $w^{*} B(X)$, then $\tilde{u}$ is also a homomorphism.

ProOF. Let $j:\left(X_{*} \widehat{\otimes} X\right) \hookrightarrow\left(X_{*} \widehat{\otimes} X\right)^{* *}$ be the canonical injection and consider its adjoint $p=j^{*}: B(X)^{* *} \rightarrow B(X)$. Then set

$$
\tilde{u}=p \circ u^{* *}: C(K)^{* *} \longrightarrow B(X) .
$$

By construction, $\tilde{u}$ is $w^{*}$-continuous and extends $u$. The equality $\|\tilde{u}\|=\|u\|$ is clear.

Assume now that $u$ is a homomorphism and that $u$ takes values in $w^{*} B(X)$. Let $\nu, \xi \in C(K)^{* *}$ and let $\left(f_{\alpha}\right)_{\alpha}$ and $\left(g_{\beta}\right)_{\beta}$ be bounded nets in $C(K) w^{*}$-converging to $v$ and $\xi$, respectively. By Lemma 2.2, we have the following equalities, where limits are taken in the $w^{*}$-topology of either $C(K)^{* *}$ or $B(X)$ :

$$
\begin{aligned}
\tilde{u}(\nu \xi) & =\widetilde{u}\left(\lim _{\alpha} \lim _{\beta} f_{\alpha} g_{\beta}\right)=\lim _{\alpha} \lim _{\beta} u\left(f_{\alpha} g_{\beta}\right)=\lim _{\alpha} \lim _{\beta} u\left(f_{\alpha}\right) u\left(g_{\beta}\right) \\
& =\lim _{\alpha} u\left(f_{\alpha}\right) \tilde{u}(\xi)=\widetilde{u}(\nu) \tilde{u}(\xi) .
\end{aligned}
$$

We refer, for example, to [17, Lemma 2.4] for the following fact.

LemMa 2.4. Consider $\tau \subseteq B(X)$ and set $\tau^{* *}=\left\{T^{* *}: T \in \tau\right\} \subseteq B\left(X^{* *}\right)$. Then $\tau$ is $R$-bounded if and only if $\tau^{* *}$ is $R$-bounded, and in this case

$$
R(\tau)=R\left(\tau^{* *}\right)
$$

For any $F \in C(K ; B(X))$, we set

$$
R(F)=R(\{F(t): t \in K\}) .
$$

Note that $R(F)$ may be infinite. If $F=\sum_{k} f_{k} \otimes b_{k}$ belongs to the algebraic tensor product $C(K) \otimes B(X)$, we set

$$
\left\|\sum_{k} f_{k} \otimes b_{k}\right\|_{R}=R(F)=R\left(\left\{\sum_{k} f_{k}(t) b_{k}: t \in K\right\}\right) .
$$

Note that, by (1.4),

$$
\|f \otimes b\|_{R} \leq 2\|f\|_{\infty}\|b\| \quad \forall f \in C(K), b \in B(X) .
$$

From this it is easy to check that $\|\cdot\|_{R}$ is finite and is a norm on $C(K) \otimes B(X)$. 
Whenever $E \subseteq B(X)$ is a closed subspace, we let

$$
C(K) \stackrel{R}{\otimes} E
$$

denote the completion of $C(K) \otimes E$ for the norm $\|\cdot\|_{R}$.

REMARK 2.5. Clearly $\|\cdot\|_{\infty} \leq\|\cdot\|_{R}$ on $C(K) \otimes B(X)$, since the $R$-bound of a set is greater than its uniform bound. Hence the canonical embedding of $C(K) \otimes B(X)$ into $C(K ; B(X))$ extends uniquely to a contraction

$$
J: C(K) \stackrel{R}{\otimes} B(X) \longrightarrow C(K ; B(X)) .
$$

Moreover, $J$ is one-to-one and, for all $\varphi \in C(K) \stackrel{R}{\otimes} B(X)$, we have $R(J(\varphi))=\|\varphi\|_{R}$. To see this, let $\left(F_{n}\right)_{n \geq 1}$ be a sequence in $C(K) \otimes B(X)$ such that $\left\|F_{n}-\varphi\right\|_{R} \rightarrow 0$ and let $F=J(\varphi)$. Then $\left\|F_{n}\right\|_{R} \rightarrow\|\varphi\|_{R}$ and $\left\|F_{n}-F\right\|_{\infty} \rightarrow 0$. According to the definition of the $R$-bound, the latter property implies that $\left\|F_{n}\right\|_{R} \rightarrow\|F\|_{R}$, which yields the result.

THEOREM 2.6. Let $u: C(K) \rightarrow B(X)$ be a bounded homomorphism.

(1) For all finite families $\left(f_{k}\right)_{k}$ in $C(K)$ and $\left(b_{k}\right)_{k}$ in $E_{u}$,

$$
\left\|\sum_{k} u\left(f_{k}\right) b_{k}\right\| \leq\|u\|^{2}\left\|\sum_{k} f_{k} \otimes b_{k}\right\|_{R} .
$$

(2) There is a (necessarily unique) bounded linear map

$$
\widehat{u}: C(K) \stackrel{R}{\otimes} E_{u} \longrightarrow B(X)
$$

such that $\widehat{u}(f \otimes b)=u(f) b$ for all $f \in C(K)$ and all $b \in E_{u}$. Furthermore, $\|\widehat{u}\| \leq\|u\|^{2}$.

PROOF. Part (2) clearly follows from part (1). To prove part (1) we introduce

$$
w: C(K) \longrightarrow B\left(X^{* *}\right), \quad w(f)=u(f)^{* *} .
$$

Then $w$ is a bounded homomorphism and $\|w\|=\|u\|$. We let $\widetilde{w}: C(K)^{* *} \rightarrow B\left(X^{* *}\right)$ be its $w^{*}$-continuous extension given by Lemma 2.3. Note that $w$ takes values in $w^{*} B\left(X^{* *}\right)$, so $\widetilde{w}$ is a homomorphism. We claim that

$$
\left\{b^{* *}: b \in E_{u}\right\} \subseteq E_{\widetilde{w}} .
$$

Indeed, let $b \in E_{u}$. Then, for all $f \in C(K)$,

$$
b^{* *} w(f)=(b u(f))^{* *}=(u(f) b)^{* *}=w(f) b^{* *} .
$$


Next, for all $v \in C(K)^{* *}$, let $\left(f_{\alpha}\right)_{\alpha}$ be a bounded net in $C(K)$ which converges to $v$ in the $w^{*}$-topology. Then, by Lemma 2.2,

$$
b^{* *} \widetilde{w}(v)=\lim _{\alpha} b^{* *} w\left(f_{\alpha}\right)=\lim _{\alpha} w\left(f_{\alpha}\right) b^{* *}=\widetilde{w}(\nu) b^{* *},
$$

and the claim follows.

Now fix $f_{1}, \ldots, f_{n} \in C(K)$ and $b_{1}, \ldots, b_{n} \in E_{u}$. For each $m \in \mathbb{N}$, there is a finite family $\left(t_{1}, \ldots, t_{N}\right)$ of $K$ and a measurable partition $\left(B_{1}, \ldots, B_{N}\right)$ of $K$ such that

$$
\left\|f_{k}-\sum_{l=1}^{N} f_{k}\left(t_{l}\right) \chi_{B_{l}}\right\|_{\infty} \leq \frac{1}{m} \quad \forall k \in\{1, \ldots, n\} .
$$

We set $f_{k}^{(m)}=\sum_{l=1}^{N} f_{k}\left(t_{l}\right) \chi_{B_{l}}$. Let $\psi: \ell_{N}^{\infty} \rightarrow \mathcal{B}^{\infty}(K)$ be defined by

$$
\psi\left(\alpha_{1}, \ldots, \alpha_{N}\right)=\sum_{l=1}^{N} \alpha_{l} \chi_{B_{l}}
$$

Then $\psi$ is a norm 1 homomorphism. According to (2.1), we can consider the bounded homomorphism

$$
\widetilde{w} \circ \psi: \ell_{N}^{\infty} \longrightarrow B\left(X^{* *}\right) .
$$

Applying Proposition 2.1 to that homomorphism, together with the above claim and Lemma 2.4, we find that

$$
\begin{aligned}
\left\|\sum_{k} \tilde{w}\left(f_{k}^{(m)}\right) b_{k}{ }^{* *}\right\| & =\left\|\sum_{k, l} f_{k}\left(t_{l}\right) \tilde{w} \circ \psi\left(e_{l}\right) b_{k}{ }^{* *}\right\| \\
& \leq\|\tilde{w} \circ \psi\|^{2} R\left(\left\{\sum_{k} f_{k}\left(t_{l}\right) b_{k}{ }^{* *}: 1 \leq l \leq N\right\}\right) \\
& \leq\|u\|^{2} R\left(\left\{\sum_{k} f_{k}(t) b_{k}{ }^{* *}: t \in K\right\}\right) \\
& \leq\|u\|^{2}\left\|\sum_{k} f_{k} \otimes b_{k}\right\|_{R} .
\end{aligned}
$$

Since $\left\|f_{k}^{(m)}-f_{k}\right\|_{\infty} \rightarrow 0$ for all $k$

$$
\left\|\sum_{k} \tilde{w}\left(f_{k}^{(m)}\right) b_{k}^{* *}\right\| \longrightarrow\left\|\sum_{k} w\left(f_{k}\right) b_{k}^{* *}\right\|=\left\|\sum_{k} u\left(f_{k}\right) b_{k}\right\|,
$$

and the result follows at once.

The following notion is implicit in several recent papers on functional calculi (see, in particular, $[8,21])$. 
Definition 2.7. Let $Z$ be a Banach space and let $v: Z \rightarrow B(X)$ be a bounded map. We set

$$
R(v)=R(\{v(z): z \in Z,\|z\| \leq 1\}),
$$

and we say that $v$ is $R$-bounded if $R(v)<\infty$.

Corollary 2.8. Suppose that $u: C(K) \rightarrow B(X)$ is a bounded homomorphism and that $v: Z \rightarrow B(X)$ is an $R$-bounded map. Assume further that $u(f) v(z)=v(z) u(f)$ for all $f \in C(K)$ and all $z \in Z$. Then there exists a (necessarily unique) bounded linear map

$$
u \cdot v: C(K ; Z) \longrightarrow B(X)
$$

such that $u \cdot v(f \otimes z)=u(f) v(z)$ for all $f \in C(K)$ and all $z \in Z$. Moreover, we have

$$
\|u \cdot v\| \leq\|u\|^{2} R(v) .
$$

Proof. Consider any finite families $\left(f_{k}\right)_{k}$ in $C(K)$ and $\left(z_{k}\right)_{k}$ in $Z$ and observe that

$$
\left\|\sum_{k} f_{k} \otimes v\left(z_{k}\right)\right\|_{R}=R\left(\left\{v\left(\sum_{k} f_{k}(t) z_{k}\right): t \in K\right\}\right) \leq R(v)\left\|\sum_{k} f_{k} \otimes z_{k}\right\|_{\infty} .
$$

Then, from Theorem 2.6 and the assumption that $v$ takes values in $E_{u}$, we find that

$$
\left\|\sum_{k} u\left(f_{k}\right) v\left(z_{k}\right)\right\| \leq\|u\|^{2} R(v)\left\|\sum_{k} f_{k} \otimes z_{k}\right\|_{\infty},
$$

which proves the result.

REMARK 2.9. As a special case of Corollary 2.8, we obtain the following result due to de Pagter and Ricker [8, Proposition 2.27]: let $K_{1}, K_{2}$ be two compact sets, and let

$$
u: C\left(K_{1}\right) \longrightarrow B(X) \text { and } v: C\left(K_{2}\right) \longrightarrow B(X)
$$

be two bounded homomorphisms which commute, that is, $u(f) v(g)=v(g) u(f)$ for all $f \in C\left(K_{1}\right)$ and $g \in C\left(K_{2}\right)$. Assume further that $R(v)<\infty$. Then there exists a bounded homomorphism

$$
w: C\left(K_{1} \times K_{2}\right) \longrightarrow B(X)
$$

such that $w_{\mid C\left(K_{1}\right)}=u$ and $w_{\mid C\left(K_{2}\right)}=v$, where $C\left(K_{j}\right)$ is regarded to be a subalgebra of $C\left(K_{1} \times K_{2}\right)$ in the natural way.

\section{Uniformly bounded $\boldsymbol{H}^{\infty}$-calculus}

We briefly recall the basic notions on $H^{\infty}$-calculus for sectorial operators. For more information, we refer, for example, to [6, 21, 23, 24]. 
For all $\theta \in(0,2 \pi)$, we define

$$
\Sigma_{\theta}=\left\{r e^{i \phi}: r>0,|\phi|<\theta\right\}
$$

and $H^{\infty}\left(\Sigma_{\theta}\right)$ to be the set of all bounded analytic functions from $\Sigma_{\theta}$ to $\mathbb{C}$. This space is equipped with the norm $\|f\|_{\infty, \theta}=\sup _{\lambda \in \Sigma_{\theta}}|f(\lambda)|$ and is a Banach algebra. We consider the auxiliary space $H_{0}^{\infty}\left(\Sigma_{\theta}\right)$ consisting of all functions $f$ in $H^{\infty}\left(\Sigma_{\theta}\right)$ for which there exist positive constants $\epsilon$ and $C$ such that

$$
|f(\lambda)| \leq C \min |\lambda|^{\epsilon},|\lambda|^{-\epsilon} \quad \forall \lambda \in \Sigma_{\theta} .
$$

A closed linear operator $A: D(A) \subseteq X \rightarrow X$ is said to be $\omega$-sectorial, where $\omega \in(0,2 \pi)$, if its domain $D(A)$ is dense in $X$, its spectrum $\sigma(A)$ is contained in $\overline{\Sigma_{\omega}}$, and for all $\theta>\omega$ there is a constant $C_{\theta}>0$ such that

$$
\left\|\lambda(\lambda-A)^{-1}\right\| \leq C_{\theta} \quad \forall \lambda \in \mathbb{C} \backslash \overline{\Sigma_{\theta}} .
$$

In this case, we define

$$
\omega(A)=\inf \{\omega: A \text { is } \omega \text {-sectorial }\} .
$$

For all $\theta \in(\omega(A), \pi)$ and all $f \in H_{0}^{\infty}\left(\Sigma_{\theta}\right)$, we define

$$
f(A)=\frac{1}{2 \pi i} \int_{\Gamma_{\gamma}} f(\lambda)(\lambda-A)^{-1} d \lambda,
$$

where $\omega(A)<\gamma<\theta$ and $\Gamma_{\gamma}$ is the boundary $\partial \Sigma_{\gamma}$ oriented counterclockwise. This definition does not depend on $\gamma$ and the resulting mapping $f \mapsto f(A)$ is an algebra homomorphism from $H_{0}^{\infty}\left(\Sigma_{\theta}\right)$ into $B(X)$. We say that $A$ has a bounded $H^{\infty}\left(\Sigma_{\theta}\right)$ calculus if the latter homomorphism is bounded, that is, if there exists a constant $C>0$ such that $\|f(A)\| \leq C\|f\|_{\infty, \theta}$ for all $f \in H_{0}^{\infty}\left(\Sigma_{\theta}\right)$. If, in addition, $A$ is one-to-one and has a dense range, then this homomorphism extends to a bounded homomorphism $H^{\infty}\left(\Sigma_{\theta}\right) \rightarrow B(X)$.

We will now focus on the sectorial operators $A$ such that $\omega(A)=0$.

Definition 3.1. We say that a sectorial operator $A$ with $\omega(A)=0$ has a uniformly bounded $H^{\infty}$-calculus if there exists a constant $C>0$ such that $\|f(A)\| \leq C\|f\|_{\infty, \theta}$ for all $\theta>0$ and $f \in H_{0}^{\infty}\left(\Sigma_{\theta}\right)$.

The space $C_{\ell}([0, \infty))$, consisting of all continuous functions $f:[0, \infty) \rightarrow \mathbb{C}$ for which $\lim _{\lambda \rightarrow \infty} f(\lambda)$ exists, is a unital commutative $C^{*}$-algebra when equipped with the natural norm

$$
\|f\|_{\infty, 0}=\sup \{|f(t)|: t \geq 0\}
$$

and involution. For all $\theta>0$, we can regard $H_{0}^{\infty}\left(\Sigma_{\theta}\right)$ as a subalgebra of $C_{\ell}([0, \infty))$, by identifying $f \in H_{0}^{\infty}\left(\Sigma_{\theta}\right)$ with its restriction $f_{\mid[0, \infty)}$. 
For all $\lambda \in \mathbb{C} \backslash[0, \infty)$, we let $R_{\lambda} \in C_{\ell}([0, \infty))$ be defined by $R_{\lambda}(t)=(\lambda-t)^{-1}$. Then we let $\mathcal{R}$ be the unital algebra generated by the $R_{\lambda}$. Equivalently, $\mathcal{R}$ is the algebra of all rational functions of nonpositive degree, whose poles lie outside the half line $[0, \infty)$. We recall that, for all $f \in H_{0}^{\infty}\left(\Sigma_{\theta}\right) \cap \mathcal{R}$, the definition of $f(A)$ given by (3.1) coincides with the usual rational functional calculus.

The following lemma is closely related to [22, Corollary 6.9].

Lemma 3.2. Let $A$ be a sectorial operator on $X$ with $\omega(A)=0$. The following assertions are equivalent.

(a) A has a uniformly bounded $H^{\infty}$-calculus.

(b) There exists a (necessarily unique) bounded unital homomorphism

$$
u: C_{\ell}([0, \infty)) \longrightarrow B(X)
$$

such that $u\left(R_{\lambda}\right)=(\lambda-A)^{-1}$ for all $\lambda \in \mathbb{C} \backslash[0, \infty)$.

Proof. Assume (a). We claim that, for all $\theta>0$ and all $f \in H_{0}^{\infty}\left(\Sigma_{\theta}\right)$,

$$
\|f(A)\| \leq C\|f\|_{\infty, 0} .
$$

Indeed, if $0 \neq f \in H_{0}^{\infty}\left(\Sigma_{\theta_{0}}\right)$ for some $\theta_{0}>0$, then there exists some $t_{0}>0$ such that $f\left(t_{0}\right) \neq 0$. Now take $r$ and $R$ such that $r<R$ and $|f(z)|<\left|f\left(t_{0}\right)\right|$ when $|z|<r$ or $|z|>R$. Choose, for every $n \in \mathbb{N}$, a $t_{n} \in \Sigma_{\theta_{0} / n}$ such that $\left|f\left(t_{n}\right)\right|=\|f\|_{\infty, \theta_{0} / n}$. Necessarily, $\left|t_{n}\right| \in[r, R]$, and there exists a convergent subsequence $t_{n_{k}}$ whose limit $t_{\infty}$ is real. Then

$$
\|f\|_{\infty, 0} \geq\left|f\left(t_{\infty}\right)\right| \geq \liminf _{\theta \rightarrow 0}\|f\|_{\infty, \theta} \geq C^{-1}\|f(A)\| .
$$

This readily implies that the rational functional calculus $\left(\mathcal{R},\|\cdot\|_{\infty, 0}\right) \rightarrow B(X)$ is bounded. By the Stone-Weierstrass theorem, this extends continuously to $C_{\ell}([0, \infty))$, which yields (b). The uniqueness property is clear.

Assume (b). Then for all $\theta \in(0, \pi)$ and all $f \in H_{0}^{\infty}\left(\Sigma_{\theta}\right) \cap \mathcal{R}$,

$$
\|f(A)\| \leq\|u\|\|f\|_{\infty, \theta} .
$$

By [24, Proposition 2.10] and its proof, this implies that $A$ has a bounded $H^{\infty}\left(\Sigma_{\theta}\right)$ calculus, with a boundedness constant uniform in $\theta$.

REMARK 3.3. An operator $A$ which admits a bounded $H^{\infty}\left(\Sigma_{\theta}\right)$-calculus for all $\theta>0$ does not necessarily have a uniformly bounded $H^{\infty}$-calculus. To get a simple example, consider

$$
A=\left(\begin{array}{ll}
1 & 1 \\
0 & 1
\end{array}\right): \ell_{2}^{2} \longrightarrow \ell_{2}^{2}
$$

Then $\sigma(A)=\{1\}$ and, for all $\theta>0$ and all $f \in H_{0}^{\infty}\left(\Sigma_{\theta}\right)$,

$$
f(A)=\left(\begin{array}{cc}
f(1) & f^{\prime}(1) \\
0 & f(1)
\end{array}\right) .
$$


Assume that $\theta<\pi / 2$. Using Cauchy's formula, it is easy to see that $\left|f^{\prime}(1)\right| \leq$ $(\sin (\theta))^{-1}\|f\|_{\infty, \theta}$ for all $f \in H_{0}^{\infty}\left(\Sigma_{\theta}\right)$. Thus $A$ admits a bounded $H^{\infty}\left(\Sigma_{\theta}\right)$-calculus.

Now let $h$ be a fixed function in $H_{0}^{\infty}\left(\Sigma_{\pi / 2}\right)$ such that $h(1)=1$, set $g_{s}(\lambda)=\lambda^{i s}$ for all $s>0$, and let $f_{s}=h g_{s}$. Then $\left\|g_{s}\right\|_{\infty, 0}=1$, and hence $\left\|f_{s}\right\|_{\infty, 0} \leq\|h\|_{\infty, 0}$ for all $s>0$. Furthermore, $g_{s}^{\prime}(\lambda)=i s \lambda^{i s-1}$ and $f_{s}^{\prime}=h^{\prime} g_{s}+h g_{s}^{\prime}$. Hence $f_{s}^{\prime}(1)=h^{\prime}(1)+i s$. Thus

$$
\left\|f_{s}(A)\right\|\left\|f_{s}\right\|_{\infty, 0}^{-1} \geq\left|f_{s}^{\prime}(1)\right|\left\|h_{s}\right\|_{\infty, 0}^{-1} \longrightarrow \infty
$$

when $s \rightarrow \infty$. Hence $A$ does not have a uniformly bounded $H^{\infty}$-calculus.

The above result can also be deduced from Proposition 3.7 below. In fact we will show in that proposition and in Corollary 3.11 that operators with a uniformly bounded $H^{\infty}$-calculus are 'rare'.

We now turn to the so-called generalized (or operator-valued) $H^{\infty}$-calculus. Throughout, we let $A$ be a sectorial operator. We let $E_{A} \subseteq B(X)$ denote the commutant of $A$, defined as the subalgebra of all bounded operators $T: X \rightarrow X$ such that $T(\lambda-A)^{-1}=(\lambda-A)^{-1} T$ for all $\lambda$ belonging to the resolvent set of $A$. We let $H_{0}^{\infty}\left(\Sigma_{\theta} ; B(X)\right)$ be the algebra of all bounded analytic functions $F: \Sigma_{\theta} \rightarrow B(X)$ for which there exist $\epsilon, C>0$ such that $\|F(\lambda)\| \leq C \min \left(|\lambda|^{\epsilon},|\lambda|^{-\epsilon}\right)$ for all $\lambda \in \Sigma_{\theta}$. Also, we let $H_{0}^{\infty}\left(\Sigma_{\theta} ; E_{A}\right)$ denote the space of all $E_{A}$-valued functions belonging to $H_{0}^{\infty}\left(\Sigma_{\theta} ; B(X)\right)$. The generalized $H^{\infty}$-calculus of $A$ is an extension of (3.1) to this class of functions. Namely, for all $F \in H_{0}^{\infty}\left(\Sigma_{\theta} ; E_{A}\right)$, we set

$$
F(A)=\frac{1}{2 \pi i} \int_{\Gamma_{\gamma}} F(\lambda)(\lambda-A)^{-1} d \lambda,
$$

where $\gamma \in(\omega(A), \pi)$. Again, this definition does not depend on $\gamma$ and the mapping $F \mapsto F(A)$ is an algebra homomorphism. The following fundamental result is due to Kalton and Weis.

Theorem 3.4 [21, Theorem 4.4], [23, Theorem 12.7]. Let $\omega_{0} \geq \omega(A)$ and assume that $A$ has a bounded $H^{\infty}\left(\Sigma_{\theta}\right)$-calculus for all $\theta>\omega_{0}$. Then, for all $\theta>\omega_{0}$, there exists a constant $C_{\theta}>0$ such that, for all $F \in H_{0}^{\infty}\left(\Sigma_{\theta} ; E_{A}\right)$,

$$
\|F(A)\| \leq C_{\theta} R\left(\left\{F(z): z \in \Sigma_{\theta}\right\}\right) .
$$

Our aim is to prove a version of this result in the case when $A$ has a uniformly bounded $H^{\infty}$-calculus. We will find in Theorem 3.6 that in this case the constant $C_{\theta}$ in (3.2) can be taken to be independent of $\theta$.

The algebra $C_{\ell}([0, \infty))$ is a $C(K)$-space and we will apply the results of Section 2 to the bounded homomophism $u$ appearing in Lemma 3.2. We recall Remark 2.5.

LEMma 3.5. Let $J: C_{\ell}([0, \infty)) \otimes^{R} B(X) \rightarrow C_{\ell}([0, \infty) ; B(X))$ be the canonical embedding. Let $\theta \in(0, \pi)$, let $F \in H_{0}^{\infty}\left(\Sigma_{\theta} ; B(X)\right)$, and let $\gamma \in(0, \theta)$. 
(1) The integral

$$
\varphi_{F}=\frac{1}{2 \pi i} \int_{\Gamma_{\gamma}} R_{\lambda} \otimes F(\lambda) d \lambda
$$

is absolutely convergent in $C_{\ell}([0, \infty)) \otimes^{R} B(X)$, and $J\left(\varphi_{F}\right)$ is equal to the restriction of $F$ to $[0, \infty)$.

(2) The set $\{F(t): t>0\}$ is $R$-bounded.

PROOF. Part (2) readily follows from part (1) and Remark 2.5. To prove part (1), observe that, for all $\lambda \in \partial \Sigma_{\gamma}$,

$$
\left\|R_{\lambda} \otimes F(\lambda)\right\|_{R} \leq 2\left\|R_{\lambda}\right\|_{\infty, 0}\|F(\lambda)\| \leq \frac{2}{\sin (\gamma)|\lambda|}\|F(\lambda)\|
$$

by (2.2). Thus, for appropriate constants $\epsilon, C>0$,

$$
\left\|R_{\lambda} \otimes F(\lambda)\right\|_{R} \leq \frac{2 C}{\sin (\gamma)} \min \left(|\lambda|^{\epsilon-1},|\lambda|^{-\epsilon-1}\right) .
$$

This shows that the integral defining $\varphi_{F}$ is absolutely convergent. Next, for all $t>0$,

$$
\left[J\left(\varphi_{F}\right)\right](t)=\frac{1}{2 \pi i} \int_{\Gamma_{\gamma}}\left(R_{\lambda} \otimes F(\lambda)\right)(t) d \lambda=\frac{1}{2 \pi i} \int_{\Gamma_{\gamma}} \frac{F(\lambda)}{\lambda-t} d \lambda=F(t)
$$

by Cauchy's theorem.

THEOREM 3.6. Let $A$ be a sectorial operator such that $\omega(A)=0$ and assume that $A$ has a uniformly bounded $H^{\infty}$-calculus. Then there exists a constant $C>0$ such that, for all $\theta>0$ and all $F \in H_{0}^{\infty}\left(\Sigma_{\theta} ; E_{A}\right)$,

$$
\|F(A)\| \leq C R(\{F(t): t>0\}) .
$$

Proof. Let $u: C_{\ell}([0, \infty)) \rightarrow B(X)$ be the representation given by Lemma 3.2. It is plain that $E_{u}=E_{A}$. Then we let

$$
\widehat{u}: C_{\ell}([0, \infty)) \stackrel{R}{\otimes} E_{A} \longrightarrow B(X)
$$

be the associated bounded map provided by Theorem 2.6.

Let $F \in H_{0}^{\infty}\left(\Sigma_{\theta} ; E_{A}\right)$ for some $\theta>0$, and let $\varphi_{F} \in C_{\ell}([0, \infty)) \otimes^{R} E_{A}$ be defined by (3.3). We claim that

$$
F(A)=\widehat{u}\left(\varphi_{F}\right)
$$

Indeed, for all $\lambda \in \partial \Sigma_{\gamma}$, we have $u\left(R_{\lambda}\right)=(\lambda-A)^{-1}$, and hence $\widehat{u}\left(R_{\lambda} \otimes F(\lambda)\right)=$ $(\lambda-A)^{-1} F(\lambda)$. Thus according to the definition of $\varphi_{F}$ and the continuity of $\widehat{u}$,

$$
\widehat{u}\left(\varphi_{F}\right)=\frac{1}{2 \pi i} \int_{\Gamma_{\gamma}} \widehat{u}\left(R_{\lambda} \otimes F(\lambda)\right) d \lambda=\frac{1}{2 \pi i} \int_{\Gamma_{\gamma}}(\lambda-A)^{-1} F(\lambda) d \lambda=F(A) .
$$

Consequently,

$$
\|F(A)\| \leq\|\widehat{u}\|\left\|\varphi_{F}\right\|_{R} \leq\|u\|^{2}\left\|\varphi_{F}\right\|_{R} .
$$

It follows from Lemma 3.5 and Remark 2.5 that $\left\|\varphi_{F}\right\|_{R}=R(\{F(t): t>0\})$, and the result follows at once. 
In the rest of this section we will investigate further the operators with a uniformly bounded $H^{\infty}$-calculus. We start with the case when $X$ is a Hilbert space.

Proposition 3.7. Let $H$ be a Hilbert space and let $A$ be a sectorial operator on $H$, such that $\omega(A)=0$. Then A admits a uniformly bounded $H^{\infty}$-calculus if and only if there exists an isomorphism $S: H \rightarrow H$ such that $S^{-1} A S$ is self-adjoint.

Proof. Assume that $A$ admits a uniformly bounded $H^{\infty}$-calculus and denote the associated representation by $u: C_{\ell}([0, \infty)) \rightarrow B(H)$. According to [28, Theorems 9.1 and 9.7], there exists an isomorphism $S: H \rightarrow H$ such that the unital homomorphism $u_{S}: C_{\ell}([0, \infty)) \rightarrow B(H)$ defined by $u_{S}(f)=S^{-1} u(f) S$ satisfies $\left\|u_{S}\right\| \leq 1$. We let $B=S^{-1} A S$. For each $s \in \mathbb{R}^{*}$, we have $\left\|R_{i s}\right\|_{\infty, 0}=|s|$ and furthermore $u_{S}\left(R_{i s}\right)=$ $S^{-1}(i s-A)^{-1} S=(i s-B)^{-1}$. Hence

$$
\left\|(i s-B)^{-1}\right\| \leq|s| \quad \forall s \in \mathbb{R}^{*} .
$$

By the Hille-Yosida theorem, this implies that $i B$ and $-i B$ both generate contractive $c_{0}$-semigroups on $H$. Thus $i B$ generates a unitary $c_{0}$-group. By Stone's theorem, this implies that $B$ is self-adjoint.

The converse implication is clear.

In the non-Hilbertian setting, we will first show that operators with a uniformly bounded $H^{\infty}$-calculus satisfy a spectral mapping theorem with respect to continuous functions defined on the one-point compactification of $\sigma(A)$. Then we will discuss the connections with spectral measures and scalar-type operators. We mainly refer to [13, Chs. 5-7] for this topic.

For any compact set $K$ and any closed subset $F \subseteq K$, we let

$$
I_{F}=\left\{f \in C(K): f_{\mid F}=0\right\} .
$$

We recall that the restriction map $f \mapsto f_{\mid F}$ induces a $*$-isomorphism $C(K) / I_{F} \rightarrow$ $C(F)$.

LEMMA 3.8. Let $K \subset \mathbb{C}$ be a compact set and let $u: C(K) \rightarrow B(X)$ be $a$ representation. Let $\kappa \in C(K)$ be the function defined by $\kappa(z)=z$ and take $T=u(\kappa)$.

(1) Then $\sigma(T) \subseteq K$ and $u$ vanishes on $I_{\sigma(T)}$.

Let $v: C(\sigma(T)) \simeq C(K) / I_{\sigma(T)} \longrightarrow B(X)$ be the representation induced by $u$.

(2) For any $f \in C(\sigma(T))$, we have $\sigma(v(f))=f(\sigma(T))$.

(3) $v$ is an isomorphism onto its range.

ProOf. The inclusion $\sigma(T) \subseteq K$ is clear. Indeed, for all $\lambda \notin K$, we have that $(\lambda-T)^{-1}$ is equal to $u\left((\lambda-\cdot)^{-1}\right)$. We will now show that $u$ vanishes on $I_{\sigma(T)}$.

Define $w: C(K) \rightarrow B\left(X^{*}\right)$ by $w(f)=[u(f)]^{*}$, and let $\widetilde{w}: C(K)^{* *} \rightarrow B\left(X^{*}\right)$ be its $w^{*}$-extension. Since $w$ takes values in $w^{*} B\left(X^{*}\right) \simeq B(X)$, this is a representation 
(see Lemma 2.3). Let $\Delta_{K}$ be the set of all Borel subsets of $K$. It is easy to check that the mapping

$$
P: \Delta_{K} \longrightarrow B\left(X^{*}\right), \quad P(B)=\widetilde{w}\left(\chi_{B}\right),
$$

is a spectral measure of class $\left(\Delta_{K}, X\right)$ in the sense of [13, p. 119]. According to [13, Proposition 5.8], the operator $T^{*}$ is prespectral of class $X$ (in the sense of [13, Definition 5.5]) and the above mapping $P$ is its resolution of the identity. Applying [13, Lemma 5.6] and the equality $\sigma\left(T^{*}\right)=\sigma(T)$, we find that $\widetilde{w}\left(\chi_{\sigma(T)}\right)=$ $P(\sigma(T))=I_{X^{*}}$. Therefore, for all $f \in I_{\sigma(T)}$,

$$
u(f)^{*}=\widetilde{w}\left(f\left(1-\chi_{\sigma(T)}\right)\right)=\widetilde{w}(f) \widetilde{w}\left(1-\chi_{\sigma(T)}\right)=0 .
$$

Hence $u$ vanishes on $I_{\sigma(T)}$.

The proofs of parts (2) and (3) now follow from [13, Proposition 5.9] and the above proof.

In what follows we consider a sectorial operator $A$ such that $\omega(A)=0$. This implies that $\sigma(A) \subseteq[0, \infty)$. By $C_{\ell}(\sigma(A))$, we denote either the space $C(\sigma(A))$ if $A$ is bounded, or the space $\left\{f: \sigma(A) \rightarrow \mathbb{C} \mid f\right.$ is continuous and $\lim _{t \rightarrow \infty} f(t)$ exists $\}$ if $A$ is unbounded. In this case, $C_{\ell}(\sigma(A))$ coincides with the space of continuous functions on the one-point compactification of $\sigma(A)$. The following strengthens Lemma 3.2.

Proposition 3.9. Let $A$ be a sectorial operator on $X$ with $\omega(A)=0$. The following assertions are equivalent.

(1) A has a uniformly bounded $H^{\infty}$-calculus.

(2) There exists a (necessarily unique) bounded unital homomorphism

$$
\Psi: C_{\ell}(\sigma(A)) \longrightarrow B(X)
$$

such that $\Psi\left((\lambda-\cdot)^{-1}\right)=(\lambda-A)^{-1}$ for all $\lambda \in \mathbb{C} \backslash \sigma(A)$.

In this case, $\Psi$ is an isomorphism onto its range and, for all $f \in C_{\ell}(\sigma(A))$,

$$
\sigma(\Psi(f))=f(\sigma(A)) \cup f_{\infty}
$$

where $f_{\infty}=\emptyset$ if $A$ is bounded and $f_{\infty}=\lim _{t \rightarrow \infty} f(t)$ if $A$ is unbounded.

Proof. Assume part (1) and let $u: C_{\ell}([0, \infty)) \rightarrow B(X)$ be given by Lemma 3.2. We introduce the particular function $\phi \in C_{\ell}([0, \infty))$ defined by $\phi(t)=(1+t)^{-1}$. Consider the $*$-isomorphism

$$
\tau: C([0,1]) \longrightarrow C_{\ell}([0, \infty)), \quad \tau(g)=g \circ \phi,
$$

and set $T=(1+A)^{-1}$. We define $\kappa(z)=z$ as in Lemma 3.8, and so $(u \circ \tau)(\kappa)=T$. Let $v: C(\sigma(T)) \rightarrow B(X)$ be the resulting factorization of $u \circ \tau$. The spectral mapping theorem gives $\sigma(A)=\phi^{-1}(\sigma(T) \backslash\{0\})$ and $0 \in \sigma(T)$ if and only if $A$ is unbounded. Thus the mapping

$$
\tau_{A}: C(\sigma(T)) \longrightarrow C_{\ell}(\sigma(A))
$$


defined by $\tau_{A}(g)=g \circ \phi$ is also a $*$-isomorphism. Take $\Psi: C_{\ell}(\sigma(A)) \rightarrow B(X)$ to be $r \circ \tau_{A}^{-1}$. This is a unital bounded homomorphism. Note that $\phi^{-1}(z)=(1-z) / z$ for all $z \in(0,1]$. Then, for all $\lambda \in \mathbb{C} \backslash \sigma(A)$,

$$
\begin{aligned}
\Psi\left((\lambda-\cdot)^{-1}\right) & =v\left((\lambda-\cdot)^{-1} \circ \phi^{-1}\right)=v\left(z \mapsto\left(\lambda-\frac{1-z}{z}\right)^{-1}\right) \\
& =v\left(z \mapsto \frac{z}{(\lambda+1) z-1}\right) \\
& =T((\lambda+1) T-1)^{-1}=(\lambda-A)^{-1} .
\end{aligned}
$$

Hence $\Psi$ satisfies part (2). Its uniqueness follows from Lemma 3.2. The fact that $\Psi$ is an isomorphism onto its range and the spectral property (3.4) follow from the above construction and Lemma 3.8. Lemma 3.2 shows that (2) implies (1).

REMARK 3.10. Let $A$ be a sectorial operator with a uniformly bounded $H^{\infty}$-calculus, and let $T=(1+A)^{-1}$. It follows from Lemma 3.8 and the proof of Proposition 3.9 that there exists a representation

$$
v: C(\sigma(T)) \longrightarrow B(X)
$$

satisfying $v(\kappa)=T$ (where $\kappa(z)=z$ ), such that $\sigma(v(f))=f(\sigma(T))$ for all $f \in$ $C(\sigma(T))$ and $v$ is an isomorphism onto its range. Also, it follows from the proof of Lemma 3.8 that $T^{*}$ is a scalar-type operator of class $X$, in the sense of [13, Definition 5.14].

Next, according to [13, Theorem 6.24], the operator $T$ (and hence $A$ ) is a scalar-type spectral operator if and only if, for all $x \in X$, the mapping $C(\sigma(T)) \rightarrow X$ taking $f$ to $v(f) x$ for all $f \in C(\sigma(T))$ is weakly compact.

Corollary 3.11. Let $A$ be a sectorial operator on $X$, with $\omega(A)=0$, and assume that $X$ does not contain a copy of $c_{0}$. Then $A$ admits a uniformly bounded $H^{\infty}$. calculus if and only if it is a scalar-type spectral operator.

Proof. The 'only if' part follows from the previous remark. Indeed, if $X$ does not contain a copy of $c_{0}$, then any bounded map $C(K) \rightarrow X$ is weakly compact [10, VI, Theorem 15]. (See also [8, 31] for related approaches.) The 'if' part follows from [16, Proposition 2.7] and its proof.

\section{REMARK 3.12.}

(1) The hypothesis on $X$ in Corollary 3.11 is necessary. Namely, it follows from [11, Theorem 3.2] and its proof that if $c_{0} \subseteq X$, then there is a sectorial operator $A$ with a uniformly bounded $H^{\infty}$-calculus on $X$ which is not scalar-type spectral.

(2) An operator on a Hilbert space is scalar-type spectral if and only if it is similar to a normal operator (see [13, Ch. 7]). Thus, when $X$ is a Hilbert space, the above corollary reduces to Proposition 3.7. 


\section{Matricial $R$-boundedness}

For all integers $n \geq 1$ and all vector spaces $E$, we denote by $M_{n}(E)$ the space of $n \times$ $n$ matrices with entries in $E$. We will be concerned mostly with the cases $E=C(K)$ or $E=B(X)$. As mentioned in the introduction, we identify $M_{n}(C(K))$ with the space $C\left(K ; M_{n}\right)$ in the usual way. We now introduce a specific norm on $M_{n}(B(X))$. Namely, for all $\left[T_{i j}\right] \in M_{n}(B(X))$, we set

$$
\begin{gathered}
\left\|\left[T_{i j}\right]\right\|_{R}=\sup \left\{\left\|\sum_{i, j=1}^{n} \epsilon_{i} \otimes T_{i j}\left(x_{j}\right)\right\|_{\operatorname{Rad}(X)}: x_{1}, \ldots, x_{n} \in X,\right. \\
\left.\left\|\sum_{j=1}^{n} \epsilon_{j} \otimes x_{j}\right\|_{\operatorname{Rad}(X)} \leq 1\right\} .
\end{gathered}
$$

Clearly $\|\cdot\|_{R}$ is a norm on $M_{n}(B(X))$. Moreover, if we consider any element of $M_{n}(B(X))$ as an operator on $\ell_{n}^{2} \otimes X$ in the natural way, and if we equip the latter tensor product with the norm of $\operatorname{Rad}_{n}(X)$, we obtain an isometric identification

$$
\left(M_{n}(B(X)),\|\cdot\|_{R}\right)=B\left(\operatorname{Rad}_{n}(X)\right) .
$$

Definition 4.1. Let $u: C(K) \rightarrow B(X)$ be a bounded linear mapping. We say that $u$ is matricially $R$-bounded if there is a constant $C \geq 0$ such that, for all $n \geq 1$ and all $\left[f_{i j}\right] \in M_{n}(C(K))$,

$$
\left\|\left[u\left(f_{i j}\right)\right]\right\|_{R} \leq C\left\|\left[f_{i j}\right]\right\|_{C\left(K ; M_{n}\right)} .
$$

REMARK 4.2. The above definition obviously extends to any bounded map $E \rightarrow$ $B(X)$ defined on an operator space $E$, or more generally on any matricially normed space (see $[14,15])$. The basic observations below apply to this general case as well.

(1) In the case when $X=H$ is a Hilbert space,

$$
\left\|\sum_{j=1}^{n} \epsilon_{j} \otimes x_{j}\right\|_{\operatorname{Rad}(H)}=\left(\sum_{j=1}^{n}\left\|x_{j}\right\|^{2}\right)^{1 / 2}
$$

for all $x_{1}, \ldots, x_{n} \in H$. Consequently, writing that a mapping $u: C(K) \rightarrow B(H)$ is matricially $R$-bounded is equivalent to writing that $u$ is completely bounded (see, for example, [28]). See Section 5 for the case when $X$ is an $L^{p}$-space.

(2) The notation $\|\cdot\|_{R}$ introduced above is consistent with that considered so far in Section 2. Indeed, let $b_{1}, \ldots, b_{n}$ in $B(X)$. Then the diagonal matrix $\operatorname{Diag}\left\{b_{1}, \ldots, b_{n}\right\} \in M_{n}(B(X))$ and the tensor element $\sum_{k=1}^{n} e_{k} \otimes b_{k} \in \ell_{n}^{\infty} \otimes B(X)$ satisfy

$$
\left\|\operatorname{Diag}\left\{b_{1}, \ldots, b_{n}\right\}\right\|_{R}=R\left(\left\{b_{1}, \ldots, b_{n}\right\}\right)=\left\|\sum_{k=1}^{n} e_{k} \otimes b_{k}\right\|_{R} .
$$

(3) If $u: C(K) \rightarrow B(X)$ is matricially $R$-bounded (with the estimate (4.2)), then $u$ is $R$-bounded and $R(u) \leq C$. Indeed, consider $f_{1}, \ldots, f_{n}$ in the unit ball of $C(K)$. 
Then we have $\left\|\operatorname{Diag}\left\{f_{1}, \ldots, f_{n}\right\}\right\|_{C\left(K ; M_{n}\right)} \leq 1$. Hence, for all $x_{1}, \ldots, x_{n}$ in $X$,

$$
\begin{aligned}
\left\|\sum_{k} \epsilon_{k} \otimes u\left(f_{k}\right) x_{k}\right\|_{\operatorname{Rad}(X)} & \leq\left\|\operatorname{Diag}\left\{u\left(f_{1}\right), \ldots, u\left(f_{n}\right)\right\}\right\|_{R}\left\|\sum_{k} \epsilon_{k} \otimes x_{k}\right\|_{\operatorname{Rad}(X)} \\
& \leq C\left\|\sum_{k} \epsilon_{k} \otimes x_{k}\right\|_{\operatorname{Rad}(X)} .
\end{aligned}
$$

Let $\left(g_{k}\right)_{k \geq 1}$ be a sequence of complex-valued, independent, standard Gaussian random variables on some probability space $\Omega_{G}$. For all $x_{1}, \ldots, x_{n}$ in $X$ let

$$
\left\|\sum_{k} g_{k} \otimes x_{k}\right\|_{G(X)}=\left(\int_{\Omega_{G}}\left\|\sum_{k} g_{k}(\lambda) x_{k}\right\|_{X}^{2} d \lambda\right)^{1 / 2} .
$$

It is well known that for each scalar-valued matrix $a=\left[a_{i j}\right] \in M_{n}$,

$$
\left\|\sum_{i, j=1}^{n} a_{i j} g_{i} \otimes x_{j}\right\|_{G(X)} \leq\|a\|_{M_{n}}\left\|\sum_{j=1}^{n} g_{j} \otimes x_{j}\right\|_{G(X)},
$$

see, for example, [9, Corollary 12.17]. For all $n \geq 1$, introduce $\sigma_{n, X}: M_{n} \rightarrow$ $B\left(\operatorname{Rad}_{n}(X)\right)$ by letting

$$
\sigma_{n, X}\left(\left[a_{i j}\right]\right)=\left[a_{i j} I_{X}\right]
$$

If $X$ has finite cotype, then we have a uniform equivalence

$$
\left\|\sum_{k} \epsilon_{k} \otimes x_{k}\right\|_{\operatorname{Rad}(X)} \asymp\left\|\sum_{k} g_{k} \otimes x_{k}\right\|_{G(X)}
$$

between Rademacher and Gaussian averages on $X$ (see, for example, [9, Theorem 12.27]). In combination with (4.3), this implies that

$$
\sup _{n \geq 1}\left\|\sigma_{n, X}\right\|<\infty \text {. }
$$

Following [29] we say that $X$ has property $(\alpha)$ if there is a constant $C \geq 1$ such that, for each finite family $\left(x_{i j}\right)$ in $X$ and each finite family $\left(t_{i j}\right)$ of complex numbers,

$$
\left\|\sum_{i, j} \epsilon_{i} \otimes \epsilon_{j} \otimes t_{i j} x_{i j}\right\|_{\operatorname{Rad}(\operatorname{Rad}(X))} \leq C \sup _{i, j}\left|t_{i j}\right|\left\|\sum_{i, j} \epsilon_{i} \otimes \epsilon_{j} \otimes x_{i j}\right\|_{\operatorname{Rad}(\operatorname{Rad}(X))} .
$$

Equivalently, $X$ has property $(\alpha)$ if and only if we have a uniform equivalence

$$
\left\|\sum_{i, j} \epsilon_{i} \otimes \epsilon_{j} \otimes x_{i j}\right\|_{\operatorname{Rad}(\operatorname{Rad}(X))} \asymp\left\|\sum_{i, j} \epsilon_{i j} \otimes x_{i j}\right\|_{\operatorname{Rad}(X)},
$$

where $\left(\epsilon_{i j}\right)_{i, j \geq 1}$ is a doubly indexed family of independent Rademacher variables.

The following is a characterization of property $(\alpha)$ in terms of the $R$-boundedness of $\sigma_{n, X}$. 
LEMMA 4.3. A Banach space $X$ has property $(\alpha)$ if and only if

$$
\sup _{n \geq 1} R\left(\sigma_{n, X}\right)<\infty .
$$

Proof. Assume that $X$ has property $(\alpha)$. This implies that $X$ has finite cotype, and hence $X$ satisfies the equivalence property (4.4). Let $a(1), \ldots, a(N)$ be in $M_{n}$ and let $z_{1}, \ldots, z_{N}$ be in $\operatorname{Rad}_{n}(X)$. Let $x_{j k}$ be in $X$ such that $z_{k}=\sum_{j} \epsilon_{j} \otimes x_{j k}$ for all $k$. We consider a doubly indexed family $\left(\epsilon_{i k}\right)_{i, k \geq 1}$ as above, as well as a doubly indexed family $\left(g_{i k}\right)_{i, k \geq 1}$ of independent standard Gaussian variables. Then

$$
\sum_{k} \epsilon_{k} \otimes \sigma_{n, X}(a(k)) z_{k}=\sum_{k, i, j} \epsilon_{k} \otimes \epsilon_{i} \otimes a(k)_{i j} x_{j k} .
$$

Hence, using the properties reviewed above,

$$
\begin{aligned}
& \left\|\sum_{k} \epsilon_{k} \otimes \sigma_{n, X}(a(k)) z_{k}\right\|_{\operatorname{Rad}(\operatorname{Rad}(X))} \\
& \asymp\left\|\sum_{k, i, j} \epsilon_{i k} \otimes a(k)_{i j} x_{j k}\right\|_{\operatorname{Rad}(X)} \asymp\left\|\sum_{k, i, j} g_{i k} \otimes a(k)_{i j} x_{j k}\right\|_{G(X)} \\
& \lesssim\left\|\left(\begin{array}{ccc}
a(1) & 0 \ldots & 0 \\
0 & \ddots & 0 \\
0 & \ldots 0 & a(N)
\end{array}\right)\right\|_{M_{N n}}\left\|\sum_{k, j} g_{j k} \otimes x_{j k}\right\|_{G(X)} \\
& \lesssim \max _{k}\|a(k)\|_{M_{n}}\left\|\sum_{k, j} \epsilon_{j k} \otimes x_{j k}\right\|_{\operatorname{Rad}(X)} \\
& \lesssim \max _{k}\|a(k)\|_{M_{n}}\left\|\sum_{k, j} \epsilon_{k} \otimes \epsilon_{j} \otimes x_{j k}\right\|_{\operatorname{Rad}(\operatorname{Rad}(X))} \\
& \lesssim \max _{k}\|a(k)\|_{M_{n}}\left\|\sum_{k} \epsilon_{k} \otimes z_{k}\right\|_{\operatorname{Rad}(\operatorname{Rad}(X))} .
\end{aligned}
$$

This shows that the $\sigma_{n, X}$ are uniformly $R$-bounded.

Conversely, assume that for some constant $C \geq 1$ we have $R\left(\sigma_{n, X}\right) \leq C$ for all $n \geq 1$. Let $\left(t_{j k}\right)_{j, k} \in \mathbb{C}^{n^{2}}$ where $\left|t_{j k}\right| \leq 1$ and, for all $k=1, \ldots, n$, let $a(k) \in M_{n}$ be the diagonal matrix with entries $t_{1 k}, \ldots, t_{n k}$ on the diagonal. Then $\|a(k)\| \leq 1$ for all $k$. Hence, applying (4.6), we find that, for all $\left(x_{j k}\right)_{j, k}$ in $X^{n^{2}}$,

$$
\begin{aligned}
& \left\|\sum_{j, k} \epsilon_{k} \otimes \epsilon_{j} \otimes t_{j k} x_{j k}\right\|_{\operatorname{Rad}(\operatorname{Rad}(X))} \\
& \quad \leq R(\{a(1), \ldots, a(n)\})\left\|\sum_{j, k} \epsilon_{k} \otimes \epsilon_{j} \otimes x_{j k}\right\|_{\operatorname{Rad}(\operatorname{Rad}(X))} \\
& \quad \leq C\left\|\sum_{j, k} \epsilon_{k} \otimes \epsilon_{j} \otimes x_{j k}\right\|_{\operatorname{Rad}(\operatorname{Rad}(X))} .
\end{aligned}
$$

This means that $X$ has property $(\alpha)$. 
Proposition 4.4. Assume that $X$ has property $(\alpha)$. Then any bounded homomorphism u: $C(K) \rightarrow B(X)$ is matricially $R$-bounded.

Proof. Let $u: C(K) \rightarrow B(X)$ be a bounded homomorphism and let $w: C(K) \rightarrow$ $B\left(\operatorname{Rad}_{n}(X)\right)$ be defined by

$$
w(f)=I_{\operatorname{Rad}_{n}} \otimes u(f) .
$$

Clearly $w$ is also a bounded homomorphism, with $\|w\|=\|u\|$. Recall the identification (4.1) and note that $w(f)=\operatorname{Diag}\{u(f), \ldots, u(f)\}$ for all $f \in C(K)$. Then, for all $a=\left[a_{i j}\right] \in M_{n}$,

$$
w(f) \sigma_{n, X}(a)=\left[a_{i j} u(f)\right]=\sigma_{n, X}(a) w(f) .
$$

By Corollary 2.8 and Lemma 4.3, the resulting mapping $w \cdot \sigma_{n, X}$ satisfies

$$
\left\|w \cdot \sigma_{n, X}: C\left(K ; M_{n}\right) \longrightarrow B\left(\operatorname{Rad}_{n}(X)\right)\right\| \leq C\|u\|^{2}
$$

where $C$ does not depend on $n$. Let $E_{i j}$ denote the canonical matrix units of $M_{n}$, for $i, j=1, \ldots, n$. Consider $\left[f_{i j}\right] \in C\left(K ; M_{n}\right) \simeq M_{n}(C(K))$ and write this matrix as $\sum_{i, j} E_{i j} \otimes f_{i j}$. Then

$$
w \cdot \sigma_{n, X}\left(\left[f_{i j}\right]\right)=\sum_{i, j=1}^{n} w\left(f_{i j}\right) \sigma_{n, X}\left(E_{i j}\right)=\sum_{i, j=1}^{n} u\left(f_{i j}\right) \otimes E_{i j}=\left[u\left(f_{i j}\right)\right] .
$$

Hence $\left\|\left[u\left(f_{i j}\right)\right]\right\|_{R} \leq C\|u\|^{2}\left\|\left[f_{i j}\right]\right\|_{C\left(K ; M_{n}\right)}$, which proves that $u$ is matricially $R$ bounded.

When $X=H$ is a Hibert space, it follows from Remark 4.2(1) that the above proposition reduces to the fact that any bounded homomorphism $C(K) \rightarrow B(H)$ is completely bounded.

We also observe that by applying the above proposition together with Remark 4.2(3) we obtain the following corollary originally due to de Pagter and Ricker [8, Corollary 2.19]. Indeed, Proposition 4.4 should be regarded as a strengthening of their result.

Corollary 4.5. Assume that $X$ has property $(\alpha)$. Then any bounded homomorphism u: $C(K) \rightarrow B(X)$ is $R$-bounded.

REMARK 4.6. The above corollary is nearly optimal. Indeed, we claim that if $X$ does not have property $(\alpha)$ and if $K$ is any infinite compact set, then there exists a unital bounded homomorphism

$$
u: C(K) \longrightarrow B(\operatorname{Rad}(X))
$$

which is not $R$-bounded. 
To prove this, let $\left(z_{n}\right)_{n \geq 1}$ be an infinite sequence of distinct points in $K$ and let $u$ be defined by

$$
u(f)\left(\sum_{k \geq 1} \epsilon_{k} \otimes x_{k}\right)=\sum_{k \geq 1} f\left(z_{k}\right) \epsilon_{k} \otimes x_{k} .
$$

According to (1.4), this is a bounded unital homomorphism satisfying $\|u\| \leq 2$. Assume now that $u$ is $R$-bounded. Let $n \geq 1$ be an integer and consider families $\left(t_{i j}\right)_{i, j}$ in $\mathbb{C}^{n^{2}}$ and $\left(x_{i j}\right)_{i, j}$ in $X^{n^{2}}$. For all $i=1, \ldots, n$, there exists $f_{i} \in C(K)$ such that $\left\|f_{i}\right\|=\sup _{j}\left|t_{i j}\right|$ and $f_{i}\left(z_{j}\right)=t_{i j}$ for all $j=1, \ldots, n$. Then

$$
\sum_{i} \epsilon_{i} \otimes u\left(f_{i}\right)\left(\sum_{j} \epsilon_{j} \otimes x_{i j}\right)=\sum_{i, j} t_{i j} \epsilon_{i} \otimes \epsilon_{j} \otimes x_{i j},
$$

and hence

$$
\begin{aligned}
\left\|\sum_{i, j} t_{i j} \epsilon_{i} \otimes \epsilon_{j} \otimes x_{i j}\right\|_{\operatorname{Rad}(\operatorname{Rad}(X))} & \leq R(u) \sup _{i}\left\|f_{i}\right\|\left\|\sum_{i, j} \epsilon_{i} \otimes \epsilon_{j} \otimes x_{i j}\right\|_{\operatorname{Rad}(\operatorname{Rad}(X))} \\
& \leq R(u) \sup _{i, j}\left|t_{i j}\right|\left\|\sum_{i, j} \epsilon_{i} \otimes \epsilon_{j} \otimes x_{i j}\right\|_{\operatorname{Rad}(\operatorname{Rad}(X))} .
\end{aligned}
$$

This shows (4.5).

\section{Application to $L^{p}$-spaces and unconditional bases}

Let $X$ be a Banach lattice with finite cotype. A classical theorem of Maurey asserts that, in addition to (4.4), we have a uniform equivalence

$$
\left\|\sum_{k} \epsilon_{k} \otimes x_{k}\right\|_{\operatorname{Rad}(X)} \asymp\left\|\left(\sum_{k}\left|x_{k}\right|^{2}\right)^{1 / 2}\right\|
$$

for finite families $\left(x_{k}\right)_{k}$ of $X$ (see, for example, [9, Theorem 16.18]). Thus a bounded linear mapping $u: C(K) \rightarrow B(X)$ is matricially $R$-bounded if there is a constant $C \geq 0$ such that, for all $n \geq 1$, for all matrices $\left[f_{i j}\right] \in M_{n}(C(K))$ and for all $x_{1}, \ldots, x_{n} \in X$,

$$
\left\|\left(\sum_{i}\left|\sum_{j} u\left(f_{i j}\right) x_{j}\right|^{2}\right)^{1 / 2}\right\| \leq C\left\|\left[f_{i j}\right]\right\|_{C\left(K ; M_{n}\right)}\left\|\left(\sum_{j}\left|x_{j}\right|^{2}\right)^{1 / 2}\right\| .
$$

Mappings satisfying this property were introduced by Simard in [32] under the name of $\ell^{2}$-cb maps. In this section we will apply a factorization property of $\ell^{2}$-cb maps established in [32], in the case when $X$ is merely an $L^{p}$-space.

Throughout this section, we let $(\Omega, \mu)$ be a $\sigma$-finite measure space. By definition, a density on that space is a measurable function $g: \Omega \rightarrow(0, \infty)$ such that $\|g\|_{1}=1$. For all such functions and all $1 \leq p<\infty$, we consider the linear mapping

$$
\phi_{p, g}: L^{p}(\Omega, \mu) \longrightarrow L^{p}(\Omega, g \mu), \quad \phi_{p, g}(h)=g^{-1 / p} h,
$$


which is an isometric isomorphism. Note that $(\Omega, g \mu)$ is a probability space. Passing from $(\Omega, \mu)$ to $(\Omega, g \mu)$ by means of the maps $\phi_{p, g}$ is usually called a change of density. A classical theorem of Johnson and Jones [18] asserts that, for all bounded operators $T: L^{p}(\mu) \rightarrow L^{p}(\mu)$, there is a density $g$ on $\Omega$ such that $\phi_{p, g} \circ T \circ \phi_{p, g}^{-1}$, initially defined on $L^{p}(g \mu)$, extends to a bounded operator on $L^{2}(g \mu)$. The next statement is an analog of that result for $C(K)$-representations.

Proposition 5.1. Let $1 \leq p<\infty$ and let $u: C(K) \rightarrow B\left(L^{p}(\mu)\right)$ be a bounded homomorphism. Then there exists a density $g: \Omega \rightarrow(0, \infty)$ and a bounded homomorphism $w: C(K) \rightarrow B\left(L^{2}(g \mu)\right)$ such that

$$
\phi_{p, g} \circ u(f) \circ \phi_{p, g}^{-1}=w(f) \quad \text { for } f \in C(K),
$$

where equality holds on $L^{2}(g \mu) \cap L^{p}(g \mu)$.

Proof. Since $X=L^{p}(\mu)$ has property $(\alpha)$, the mapping $u$ is matricially $R$-bounded by Proposition 4.4. According to the above discussion, this means that $u$ is $\ell^{2}-\mathrm{cb}$ in the sense of [32, Definition 2]. The result therefore follows from [32, Theorems 3.4 and 3.6].

We will now focus on Schauder bases on separable $L^{p}$-spaces. We refer to [27, Ch. 1] for general information on this topic. We simply recall that a sequence $\left(e_{k}\right)_{k \geq 1}$ in a Banach space $X$ is a basis if, for every $x \in X$, there exists a unique scalar sequence $\left(a_{k}\right)_{k \geq 1}$ such that $\sum_{k} a_{k} e_{k}$ converges to $x$. A basis $\left(e_{k}\right)_{k \geq 1}$ is said to be unconditional if this convergence is unconditional for all $x \in X$. We record the following standard characterization.

LEMMA 5.2. A sequence $\left(e_{k}\right)_{k \geq 1} \subset X$ of nonzero vectors is an unconditional basis of $X$ if and only if $X=\overline{\operatorname{Span}}\left\{e_{k}: k \geq 1\right\}$ and there exists a constant $C \geq 1$ such that, for all bounded scalar sequences $\left(\lambda_{k}\right)_{k \geq 1}$ and for all finite scalar sequences $\left(a_{k}\right)_{k \geq 1}$,

$$
\left\|\sum_{k} \lambda_{k} a_{k} e_{k}\right\| \leq C \sup _{k}\left|\lambda_{k}\right|\left\|\sum_{k} a_{k} e_{k}\right\| .
$$

We will need the following elementary lemma.

Lemma 5.3. Let $(\Omega, v)$ be a $\sigma$-finite measure space, let $1 \leq p<\infty$ and let $Q$ : $L^{p}(v) \rightarrow L^{p}(v)$ be a finite rank bounded operator such that $Q_{\mid L^{2}(v) \cap L^{p}(v)}$ extends to a bounded operator $L^{2}(v) \rightarrow L^{2}(v)$. Then $Q\left(L^{p}(v)\right) \subset L^{2}(v)$.

Proof. Let $E=Q\left(L^{p}(v) \cap L^{2}(v)\right)$. By assumption, $E$ is a finite-dimensional subspace of $L^{p}(v) \cap L^{2}(v)$. Since $E$ is automatically closed under the $L^{p}$-norm and $Q$ is continuous, we find that $Q\left(L^{p}(v)\right)=E$.

THEOREM 5.4. Let $1 \leq p<\infty$ and assume that $\left(e_{k}\right)_{k \geq 1}$ is an unconditional basis of $L^{p}(\Omega, \mu)$. Then there exists a density $g$ on $\Omega$ such that $\phi_{p, g}\left(e_{k}\right) \in L^{2}(g \mu)$ for all $k \geq 1$, and the sequence $\left(\phi_{p, g}\left(e_{k}\right)\right)_{k \geq 1}$ is an unconditional basis of $L^{2}(g \mu)$. 
PROOF. Property (5.1) implies that, for all $\lambda=\left(\lambda_{k}\right)_{k \geq 1} \in \ell^{\infty}$, there exists a (necessarily unique) bounded operator $T_{\lambda}: L^{p}(\mu) \rightarrow L^{p}(\mu)$ such that $T_{\lambda}\left(e_{k}\right)=\lambda_{k} e_{k}$ for all $k \geq 1$. Moreover, $\left\|T_{\lambda}\right\| \leq C\|\lambda\|_{\infty}$. We can therefore consider the mapping

$$
u: \ell^{\infty} \longrightarrow B\left(L^{p}(\mu)\right), \quad u(\lambda)=T_{\lambda},
$$

and $u$ is a bounded homomorphism. By Proposition 5.1, there is a constant $C_{1}>0$ and a density $g$ on $\Omega$ such that the mapping

$$
\phi T_{\lambda} \phi^{-1}: L^{p}(g \mu) \longrightarrow L^{p}(g \mu)
$$

(where $\phi=\phi_{p, g}$ ) extends to a bounded operator

$$
S_{\lambda}: L^{2}(g \mu) \longrightarrow L^{2}(g \mu)
$$

for all $\lambda \in \ell^{\infty}$, where $\left\|S_{\lambda}\right\| \leq C_{1}\|\lambda\|_{\infty}$.

Assume first that $p \geq 2$, so that $L^{p}(g \mu) \subset L^{2}(g \mu)$. Let $\lambda=\left(\lambda_{k}\right)_{k \geq 1}$ in $\ell^{\infty}$ and let $\left(a_{k}\right)_{k \geq 1}$ be a finite scalar sequence. Then $S_{\lambda}\left(\phi\left(e_{k}\right)\right)=\phi T_{\lambda} \phi^{-1}\left(\phi\left(e_{k}\right)\right)=\lambda_{k} \phi\left(e_{k}\right)$ for all $k \geq 1$, and hence

$$
\begin{aligned}
\left\|\sum_{k} \lambda_{k} a_{k} \phi\left(e_{k}\right)\right\|_{L^{2}(g \mu)} & =\left\|S_{\lambda}\left(\sum_{k} a_{k} \phi\left(e_{k}\right)\right)\right\|_{L^{2}(g \mu)} \\
& \leq C_{1}\|\lambda\|_{\infty}\left\|\sum_{k} a_{k} \phi\left(e_{k}\right)\right\|_{L^{2}(g \mu)} .
\end{aligned}
$$

Moreover, the linear span of the $\phi\left(e_{k}\right)$ is dense in $L^{p}(g \mu)$, and hence in $L^{2}(g \mu)$. By Lemma 5.2, this shows that $\left(\phi\left(e_{k}\right)\right)_{k \geq 1}$ is an unconditional basis of $L^{2}(g \mu)$.

Assume now that $1 \leq p<2$. For all $n \geq 1$, let $f_{n} \in \ell^{\infty}$ be defined by $\left(f_{n}\right)_{k}=\delta_{n, k}$ for all $k \geq 1$, and let $Q_{n}: L^{p}(g \mu) \rightarrow L^{p}(g \mu)$ be the projection defined by

$$
Q_{n}\left(\sum_{k} a_{k} \phi\left(e_{k}\right)\right)=a_{n} \phi\left(e_{n}\right) \text {. }
$$

Then $Q_{n}=\phi T_{f_{n}} \phi^{-1}$ and hence $Q_{n}$ extends to an $L^{2}$ operator. Therefore, $\phi\left(e_{n}\right)$ belongs to $L^{2}(g \mu)$ by Lemma 5.3.

Let $p^{\prime}=p /(p-1)$ be the conjugate number of $p$, let $\left(e_{k}^{*}\right)_{k \geq 1}$ be the bi-orthogonal system of $\left(e_{k}\right)_{k \geq 1}$, and let $\phi^{\prime}=\phi^{*-1}$. (It is easy to check that $\phi^{\prime}=\phi_{p^{\prime}, g}$, but we will not use this point.) The linear span of the $e_{k}^{*}$ is $w^{*}$-dense in $L^{p^{\prime}}(\mu)$. Equivalently, the linear span of the $\phi^{\prime}\left(e_{k}^{*}\right)$ is $w^{*}$-dense in $L^{p^{\prime}}(g \mu)$, and hence it is dense in $L^{2}(g \mu)$. Moreover, for all $\lambda \in \ell^{\infty}$ and for all $k \geq 1$, we have $T_{\lambda}^{*}\left(e_{k}^{*}\right)=\lambda_{k} e_{k}^{*}$. Thus, for all finite scalar sequences $\left(a_{k}\right)_{k \geq 1}$,

$$
\sum_{k} \lambda_{k} a_{k} \phi^{\prime}\left(e_{k}^{*}\right)=\left(\phi T_{\lambda} \phi^{-1}\right)^{*}\left(\sum_{k} a_{k} \phi^{\prime}\left(e_{k}^{*}\right)\right)=S_{\lambda}^{*}\left(\sum_{k} a_{k} \phi^{\prime}\left(e_{k}^{*}\right)\right) .
$$


Hence

$$
\left\|\sum_{k} \lambda_{k} a_{k} \phi^{\prime}\left(e_{k}^{*}\right)\right\|_{L^{2}(g \mu)} \leq C_{1}\left\|\sum_{k} a_{k} \phi^{\prime}\left(e_{k}^{*}\right)\right\|_{L^{2}(g \mu)} .
$$

According to Lemma 5.2, this shows that $\left(\phi^{\prime}\left(e_{k}^{*}\right)\right)_{k \geq 1}$ is an unconditional basis of $L^{2}(g \mu)$. It is plain that $\left(\phi\left(e_{k}\right)\right)_{k \geq 1} \subset L^{2}(g \mu)$ is the bi-orthogonal system of $\left(\phi^{\prime}\left(e_{k}^{*}\right)\right)_{k \geq 1} \subset L^{2}(g \mu)$. This shows that, in turn, $\left(\phi\left(e_{k}\right)\right)_{k \geq 1}$ is an unconditional basis of $L^{2}(g \mu)$.

We will now establish a variant of Theorem 5.4 for conditional bases. Recall that if $\left(e_{k}\right)_{n \geq 1}$ is a basis on some Banach space $X$, then the projections $P_{N}: X \rightarrow X$ defined by

$$
P_{N}\left(\sum_{k} a_{k} e_{k}\right)=\sum_{k=1}^{N} a_{k} e_{k}
$$

are uniformly bounded. We will say that $\left(e_{k}\right)_{k \geq 1}$ is an $R$-basis if the set $\left\{P_{N}: N \geq 1\right\}$ is actually $R$-bounded. It follows from [4, Corollary 3.15] that any unconditional basis on $L^{p}$ is an $R$-basis. See Remark 5.6(2) for more details on this.

Proposition 5.5. Let $1 \leq p<\infty$ and let $\left(e_{k}\right)_{k \geq 1}$ be an $R$-basis of $L^{p}(\Omega, \mu)$. Then there exists a density $g$ on $\Omega$ such that $\phi_{p, g}\left(e_{k}\right) \in L^{2}(g \mu)$ for all $k \geq 1$, and the sequence $\left(\phi_{p, g}\left(e_{k}\right)\right)_{k \geq 1}$ is a basis of $L^{2}(g \mu)$.

PROOF. According to [26, Theorem 2.1], there exists a constant $C \geq 1$ and a density $g$ on $\Omega$ such that, taking $\phi=\phi_{p, g}$,

$$
\left\|\phi P_{N} \phi^{-1} h\right\|_{2} \leq C\|h\|_{2} \quad \forall N \geq 1, h \in L^{2}(g \mu) \cap L^{p}(g \mu) .
$$

Then the proof is similar to that of Theorem 5.4, using [27, Proposition 1.a.3] instead of Lemma 5.2. We skip the details.

REMARK 5.6. (1) Theorem 5.4 and Proposition 5.5 can be easily extended to finitedimensional Schauder decompositions. We refer to [27, Section 1.g] for general information on this notion. Given a Schauder decomposition $\left(X_{k}\right)_{k \geq 1}$ of a Banach space $X$, let $P_{N}$ be the associated projections; namely, for all $N \geq 1, P_{N}: X \rightarrow X$ is the bounded projection onto $X_{1} \oplus \cdots \oplus X_{N}$ vanishing on $X_{k}$ for all $k \geq N+1$. We say that $\left(X_{k}\right)_{k \geq 1}$ is an $R$-Schauder decomposition if the set $\left\{P_{N}: N \geq 1\right\}$ is $R$-bounded. Then we find that, for all $1<p<\infty$ and for all finite-dimensional $R$-Schauder (respectively unconditional) decompositions $\left(X_{k}\right)_{k \geq 1}$ of $L^{p}(\mu)$, there exists a density $g$ on $\Omega$ such that $\phi_{p, g}\left(X_{k}\right) \subset L^{2}(g \mu)$ for all $k \geq 1$, and $\left(\phi_{p, g}\left(X_{k}\right)\right)_{k \geq 1}$ is a Schauder (respectively unconditional) decomposition of $L^{2}(g \mu)$.

(2) The concept of $R$-Schauder decompositions can be tracked down to [2], and it played a key role in [4] and in various works on $L^{p}$-maximal regularity and $H^{\infty}$-calculus; see, in particular, $[20,21]$. Let $C_{p}$ denote the Schatten spaces. For $1<p \neq 2<\infty$, an explicit example of a Schauder decomposition on $L^{2}\left([0,1] ; C_{p}\right)$ 
which is not $R$-Schauder is given in [4, Section 5]. More generally, it follows from [20] that whenever a reflexive Banach space $X$ has an unconditional basis and is not isomorphic to $\ell^{2}$, then $X$ admits a finite-dimensional Schauder decomposition which is not $R$-Schauder. This applies, in particular, to $X=L^{p}([0,1])$, for all $1<p \neq 2<\infty$. However, whether $L^{p}([0,1])$ admits a Schauder basis that is not $R$-Schauder is apparently an open question.

We finally mention that, according to [21, Theorem 3.3], any unconditional decomposition on a Banach space $X$ with property $(\Delta)$ is an $R$-Schauder decomposition.

\section{Acknowledgement}

We are grateful to the referee for various remarks and references which improved the presentation of this paper.

\section{References}

[1] W. Arendt and $\mathrm{S} . \mathrm{Bu}$, 'The operator-valued Marcinkiewicz multiplier theorem and maximal regularity', Math. Z. 240 (2002), 311-343.

[2] E. Berkson and T. A. Gillespie, 'Spectral decompositions and harmonic analysis on UMD Banach spaces', Studia Math. 112 (1994), 13-49.

[3] J. W. Bunce, 'Representations of strongly amenable $C^{*}$-algebras', Proc. Amer. Math. Soc. 32 (1972), 241-246.

[4] P. Clément, B. de Pagter, F. A. Sukochev and H. Witvliet, 'Schauder decompositions and multiplier theorems', Studia Math. 138 (2000), 135-163.

[5] J. B. Conway, A Course in Operator Theory, Graduate Studies in Mathematics, 21 (American Mathematical Society, Providence, RI, 2000).

[6] M. Cowling, I. Doust, A. McIntosh and A. Yagi, 'Banach space operators with a bounded $H^{\infty}$ functional calculus', J. Aust. Math. Soc., Ser. A 60 (1996), 51-89.

[7] G. Dales, Banach Algebras and Automatic Continuity, London Mathematical Society Monographs, New Series, 24 (Oxford University Press, Oxford, 2000).

[8] B. de Pagter and W. J. Ricker, ' $C(K)$-representations and $R$-boundedness', J. London Math. Soc. 76 (2007), 498-512.

[9] J. Diestel, H. Jarchow and A. Tonge, Absolutely Summing Operators, Cambridge Studies in Advanced Mathematics, 43 (Cambridge University Press, Cambridge, 1995).

[10] J. Diestel and J. J. Uhl, Vector Measures, Mathematical Surveys and Monographs, 15 (American Mathematical Society, Providence, RI, 1977).

[11] I. Doust and R. deLaubenfels, 'Functional calculus, integral representations, and Banach space geometry’, Quaestiones Math. 17 (1994), 161-171.

[12] I. Doust and T. A. Gillespie, 'Well-boundedness of sums and products of operators', J. London Math. Soc. 68 (2003), 183-192.

[13] H. R. Dowson, Spectral Theory of Linear Operators, London Mathematical Society Monographs, 12 (Academic Press, London, 1978).

[14] E. Effros and Z.-J. Ruan, 'On matricially normed spaces', Pacific J. Math. 132 (1988), $243-264$.

[15] E. Effros and Z.-J. Ruan, Operator Spaces, London Mathematical Society Monographs, New Series, 23 (Oxford University Press, Oxford, 2000).

[16] A. M. Fröhlich and L. Weis, ' $H^{\infty}$ calculus and dilations', Bull. Soc. Math. France 134 (2006), 487-508.

[17] M. Hoffmann, N. J. Kalton and T. Kucherenko, ' $R$-bounded approximating sequences and applications to semigroups', J. Math. Anal. Appl. 294 (2004), 373-386.

[18] W. B. Johnson and L. Jones, 'Every $L^{p}$ operator is an $L^{2}$ operator', Proc. Amer. Math. Soc. 72 (1978), 309-312. 
[19] R. V. Kadison and J. R. Ringrose, Fundamentals of the Theory of Operator Algebras, I and II, Graduate Studies in Mathematics, 16 (American Mathematical Society, Providence, RI, 1997).

[20] N. J. Kalton and G. Lancien, 'A solution to the problem of $L^{p}$-maximal regularity', Math. Z. 235 (2000), 559-568.

[21] N. J. Kalton and L. Weis, 'The $H^{\infty}$-calculus and sums of closed operators', Math. Ann. 321 (2001), 319-345.

[22] N. J. Kalton and L. Weis, 'The $H^{\infty}$ functional calculus and square function estimates', Preprint.

[23] P. C. Kunstmann and L. Weis, 'Maximal $L_{p}$-regularity for parabolic equations, Fourier multiplier theorems and $H^{\infty}$-functional calculus', in: Functional Analytic Methods for Evolution Equations, Lecture Notes in Mathematics, 1855 (Springer, New York, 2004), pp. 65-311.

[24] C. Le Merdy, ' $H^{\infty}$-functional calculus and applications to maximal regularity', Publ. Math. Besançon 16 (1998), 41-77.

[25] C. Le Merdy, 'A strong similarity property of nuclear $C^{*}$-algebras', Rocky Mountain J. Math. 30 (2000), 279-292.

[26] C. Le Merdy and A. Simard, 'A factorization property of $R$-bounded sets of operators on $L^{p}$ spaces', Math. Nachr. 243 (2002), 146-155.

[27] J. Lindenstrauss and L. Tzafriri, Classical Banach Spaces I (Springer, Berlin, 1977).

[28] V. I. Paulsen, Completely Bounded Maps and Operator Algebras, Cambridge Studies in Advanced Mathematics, 78 (Cambridge University Press, Cambridge, 2002).

[29] G. Pisier, 'Some results on Banach spaces without local unconditional structure', Compositio Math. 37 (1978), 3-19.

[30] G. Pisier, Similarity Problems and Completely Bounded Maps (Second, expanded version), Lecture Notes in Mathematics, 1618 (Springer, New York, 2001).

[31] W. J. Ricker, Operator Algebras Generated by Commuting Projections: A Vector Measure Approach, Lecture Notes in Mathematics, 1711 (Springer, New York, 1999).

[32] A. Simard, 'Factorization of sectorial operators with bounded $H^{\infty}$-functional calculus', Houston J. Math. 25 (1999), 351-370.

\author{
CHRISTOPH KRIEGLER, Institut für Analysis, Karlsruhe Institute of Technology, \\ Kaiserstrasse 89, 76133 Karlsruhe, Germany \\ and \\ Laboratoire de Mathématiques, Université de Franche-Comté, \\ 25030 Besançon Cedex, France \\ e-mail: christoph.kriegler@univ-fcomte.fr
}

CHRISTIAN LE MERDY, Laboratoire de Mathématiques, Université de Franche-Comté, 25030 Besançon Cedex, France e-mail: clemerdy@univ-fcomte.fr 